\title{
A PROPERTY OF UNIVALENT FUNCTIONS IN $A_{p}$ DAVID WALSH \\ Department of Mathematics, NUI Maynooth, Co. Kildare, Ireland
}

(Received 22 June, 1998)

\begin{abstract}
The univalent functions in the diagonal Besov space $A_{p}$, where $1<p<\infty$, are characterized in terms of the distance from the boundary of a point in the image domain. Here $A_{2}$ is the Dirichlet space. A consequence is that there exist functions in $A_{p}, p>2$, for which the area of the complement of the image of the unit disc is zero.
\end{abstract}

1991 Mathematics Subject Classification. 30C99, 46E35

Introduction. The Dirichlet space $A_{2}$ consists of analytic functions on the disc whose images, counting multiplicity, have finite area. If one relaxes the condition on $f$ by allowing $f$ to belong to the somewhat larger space, the diagonal Besov space $A_{p}=A_{p p}^{1 / p}$ with $p>2$, what can one say about the area of the image of $f$ ? In this note we show that for such a function, the complement of the image of the unit disc may have zero area.

Let $1<p<\infty$. Denote by $A_{p}$ the space of functions $f(z)$ that are analytic on the open unit disc $D=\{z:|z|<1\}$, and satisfy

$$
\int_{D}\left|f^{\prime}(z)\right|^{p}\left(1-|z|^{2}\right)^{p-2} d A(z)<\infty
$$

The spaces $A_{p}$ are called the diagonal Besov spaces to distinguish them from the more general class of Besov spaces $A_{p q}^{s}$, where $s>0,1<p, q<\infty$. See [2]. If we set $s=1 / p, q=p$ we obtain the space we call $A_{p}$. If $p<r$, then $A_{p} \subset A_{r}$, while $A_{2}$ is the Dirichlet space. On letting $p$ tend to infinity we may identify $A_{\infty}$ as the space of analytic functions $f$ satisfying

$$
\left(1-|z|^{2}\right)\left|f^{\prime}(z)\right|=O(1) \text { as }|z| \rightarrow 1-.
$$

This is the Bloch space $B$. The subspace of $B$, consisting of functions $f$ for which

$$
\left(1-|z|^{2}\right)\left|f^{\prime}(z)\right| \rightarrow 0 \text { as }|z| \rightarrow 1-,
$$

is denoted by $B_{0}$, and called the little Bloch space.

The distance function $d(w)$. An analytic function $f$ on $D$ which is one to one is said to be univalent. For a point $w=f(z)$ in the image domain an important notion is that of distance to the boundary:

$$
d(w)=\inf \{|w-\zeta|, \quad \zeta \in \partial f(D)\} .
$$

We state the following corollary of Koebe's Distortion Theorem [3]. 
Theorem A. Suppose that $f$ is univalent in D. Then

$$
\frac{1}{4} d(w) \leq\left(1-|z|^{2}\right)\left|f^{\prime}(z)\right| \leq d(w) .
$$

Thus for univalent $f$, we have

(i) $f \in B$ if and only if $\sup _{w} d(w)<\infty$,

(ii) $f \in B_{0}$ if and only if $\lim _{|z| \rightarrow 1} d(w)=0$.

We can extend this result to $A_{p}$.

Theorem 1. Let $f$ be univalent in $D$ and $1<p<\infty$. Then $f \in A_{p}$ if and only if

$$
\int_{f(D)} d(w)^{p-2} d A(w)<\infty
$$

Proof. From Theorem A we have, for $p>2$,

$$
\frac{1}{4^{p-2}} d(w)^{p-2} \leq\left(1-|z|^{2}\right)^{p-2}\left|f^{\prime}(z)\right|^{p-2} \leq d(w)^{p-2} .
$$

We observe that $d A(w)=\left|f^{\prime}(z)\right|^{2} d A(z)$. Integrating the inequality above with respect to the measure $d A(w)$ over the image domain $f(D)$, we get

$$
\frac{1}{4^{p-2}} \int_{f(D)} d(w)^{p-2} d A(w) \leq \int_{D}\left(1-|z|^{2}\right)^{p-2}\left|f^{\prime}(z)\right|^{p} d A(z) \leq \int_{f(D)} d(w)^{p-2} d A(w) .
$$

For $1<p<2$, the inequalities are reversed. The result follows.

This simple result has useful consequences which we shall see in a moment. Pommerenke [4] has given a condition whereby a non-vanishing univalent function $g$ in $D$ has the property that $\log g$ belongs to $B_{0}$ (and consequently also to the space $V M O A)$.

As above, for $w=g(z)$ we let $d(w)=\inf \{|w-\zeta|, \quad \zeta \in \partial g(D)\}$. Then

$$
\log g \in B_{0} \text { if and only if } \frac{d(w)}{|w|} \rightarrow 0 \text { as }|w| \rightarrow 0, \infty
$$

We can extend this result to $A_{p}$.

Theorem 2. Suppose that $g$ is univalent and non-vanishing in $D$, where $1<p<\infty$, and let $f(z)=\log g(z)$. Then

$$
f \in A_{p} \text { if and only if } \int_{g(D)} \frac{d(w)^{p-2}}{|w|^{p}} d A(w)<\infty .
$$


Proof. As before, for $p>2$, we have

$$
\frac{1}{4^{p-2}} d(w)^{p-2} \leq\left(1-|z|^{2}\right)^{p-2}\left|g^{\prime}(z)\right|^{p-2} \leq d(w)^{p-2} .
$$

This gives

$$
\frac{1}{4^{p-2}} \frac{d(w)^{p-2}}{|w|^{p}} d A(w) \leq\left(1-|z|^{2}\right)^{p-2} \frac{\left|g^{\prime}(z)\right|^{p}}{|g(z)|^{p}} d A(z) \leq \frac{d(w)^{p-2}}{|w|^{p}} d A(w) .
$$

Observing that the middle term is $\left(1-|z|^{2}\right)^{p-2}\left|f^{\prime}(z)\right|^{p} d A(z)$ we get the result by integration. Again, for $1<p<2$ the inequalities are reversed.

Two applications of Theorem 1. According to a theorem of Richter and Shields [5], every function $f$ in the Dirichlet space $A_{2}$ can be written as the quotient of two bounded functions in $A_{2}$. This result depends on the fact that there is a compact set $K$ having positive two-dimensional Lebesgue measure lying in the complement of $f(D)$. Their proof is such that if we could prove the last statement above for any $f \in A_{p}$, then an analogue of their conclusion would hold: if $f \in A_{p}$ then $f=g / h$, where $g, h \in A_{p} \cap H^{\infty}$. We need only take $p>2$ since if $p \leq 2$ the area of $f(D)$ is finite. However we shall now show that there exists a univalent function $f \in A_{p}$ such that the complement of the image $f(D)$ has zero two-dimensional Lebesgue measure.

The following construction uses an idea from an unpublished manuscript of Douglas M. Campbell. Consider for each integer $m \geq 0$, the half-strip

$$
S_{m, 1}=\{x+i y: m<x<m+1,0<y<\infty\}
$$

We perform a countable number of operations the $n^{\text {th }}$ of which is the removal from $S_{m, 1}$ of $2^{n-1}$ infinite vertical slits whose initial points are $2 \pi i n /(m+1)^{2}+k / 2^{n}+m,\left(k=1,3, \ldots 2^{n}-1\right)$. In the lower half strip

$$
S_{m, 2}=\{x+i y: m<x<m+1,-\infty<y<0\},
$$

we carry out operations which are the mirror image of those above; that is we perform a countable number of operations the $n^{\text {th }}$ of which is the removal from $S_{m, 2}$ of $2^{n-1}$ infinite vertical slits whose initial points are $-2 \pi i n /(m+1)^{2}+k / 2^{n}+m$, $\left(k=1,3, \ldots 2^{n}-1\right)$. We have now made a countable number of slits in the right half plane. Finally we extend the slitting procedure to the left half plane by reflection in the $y$ axis. We denote the resulting simply connected domain by $G$. Note that $0 \in G$ and also each line $\Re z=m$ for each integer $m$. Now let $f$ be the conformal mapping of $D$ onto $G$ with $f(0)=0, f^{\prime}(0)>0$.

We shall now show that $\int_{G} d(w)^{p-2} d A(w)<\infty$ and invoke Theorem 1 , thereby showing that $f \in A_{p}$. We may confine attention to the first quadrant. Consider the half-strip $S_{m, 1}$ and, for $n \geq 1$, consider the subset

$$
L(m, n)=\left\{w \in S_{m, 1}: 2 \pi n /(m+1)^{2}<\Im w<2 \pi(n+1) /(m+1)^{2}\right\}
$$


with area $2 \pi /(m+1)^{2}$. It is easy to see that $d(w)<1 / 2^{n}$ for each $w \in L(m, n)$. It follows that

$$
\int_{S_{m, 1}} d(w)^{p-2} d A(w) \leq \sum_{n=0}^{\infty} \frac{1}{2^{n(p-2)}} \frac{2 \pi}{(m+1)^{2}}=2 \pi C_{p} /(m+1)^{2} .
$$

Summing over $m$ now gives the desired result. It is clear that the area of the complement of $f(D)$ is zero.

REMARK. Under the assumption $2 \leq p<\infty, 0<q<\infty$ and $0<s<1 / 2$, K. Dyakonov [1] has shown that every function in $A_{p q}^{s}$ is the ratio of two bounded functions in $A_{p q}^{s}$. We noted above that if $1<p<2$ then the proof of Richter and Shields can be adapted to give the result for $A_{p}$. Thus the conclusion holds for $A_{p}$, for all $p>1$.

For a second application suppose that $f$ is a bounded univalent function on $D$. Clearly $f \in A_{2}$, since the area of $f(D)$ is finite. We show that $f$ need not belong to $A_{p}$ for any $p<2$. Consider the open unit square $Q=\{(x, y): 0<x<1,0<y<1\}$. For each $n \geq 1$ we make $2^{n-1}$ vertical slits in $Q$ each of height $1 /(n+1)$ with base points $\left(k / 2^{n}, k=1,3, \ldots 2^{n}-1\right)$. The resulting simply connected domain is called $G$. Let $f$ be a conformal map of $D$ onto $G$ and let $w=u+i v$ be a point in $G$. Consider the points $w$ of $G$ lying in a strip $\frac{1}{n+2}<v<\frac{1}{n+1}$. We readily check that $d(w) \leq 1 / 2^{n+1}$ for all points $w$ in the strip. Choose $p<2$. It follows that

$$
\int_{G} d(w)^{p-2} d A(w) \geq 2^{2-p} \sum_{n=1}^{\infty} \frac{2^{(2-p) n}}{(n+1)(n+2)}=\infty,
$$

which implies by Theorem 1 that $f$ is not in $A_{p}$.

\section{REFERENCES}

1. K. Dyakonov, Besov spaces and outer functions, Michigan Math. J. 45 (1998), 143-157.

2. S. M. Nikolskii, Approximation of functions of several variables and imbedding theorems (Springer-Verlag, 1975).

3. Ch. Pommerenke, Univalent functions (Vandenhoeck and Ruprecht, Gottingen 1975).

4. Ch. Pommerenke, Schlichte funktionen und analytische funktionen von beschrankter mittlerer oszillation, Comment. Math. Helv. 52 (1977), 591-602.

5. S. Richter and A. Shields, Bounded analytic functions in the Dirichlet space, Math. Z. 198 (1988), 151-159. 